\title{
EXAMEN CRÍTICO DE LA BIBLIOGRAFÍA SOBRE LA HISTORIA DE LA TRADUCCIÓN EN ESPAÑA
}

\author{
David Pérez Blázquez \\ $<$ dperez@tradung.com> \\ Freelance translator
}

\begin{abstract}
Resumen
El artículo aborda de forma crítica y sucinta el recorrido de los estudios sobre historia de la traducción en España, partiendo tanto de los hitos bibliográficos como del recuento de la producción investigadora menor. Ante la dispersión temática existente y el incremento que durante las últimas décadas han experimentado estos estudios, la presente revisión historiográfica pone de manifiesto la necesidad de realizar en futuros trabajos una síntesis periodificadora y clasificadora que pueda ofrecer una perspectiva panorámica y práctica para la investigación y teorización.
\end{abstract}

\begin{abstract}
"A Critical Review of the Bibliography on the History of Translation in Spain"

This article deals critically and succinctly with the development of the studies on translation history in Spain, starting from counting the bibliographic production (research, studies, articles, publications, etc.). Given the existing thematic dispersion and the increase that these studies have experienced in the last few decades, this historiographic review highlights the need for a synthesis in future works establishing periods and classification in order to provide a panoramic and practical view to research and theorizing.
\end{abstract}

Palabras clave: Historia de la traducción. España. Bibliografía. Historiografía. Traducción.

Keywords: Translation history. Spain. Bibliography. Historiography. Translation.

Manuscript received on May 6, 2012 and accepted on October 5, 2012. 



\section{Introducción}

Su historia se pierde en el tiempo; ha sido vital en la evolución de la Humanidad, imprescindible para arrastrar las semillas del pensamiento por medio mundo, para desarrollar el comercio y las ciencias, para armar y desarmar guerras. Y, sin embargo, la traducción parece estar condenada, incluso entre quienes la practican y la enseñan, a no ser más que una actividad extremadamente técnica, efímera y utilitaria al servicio otras disciplinas más "relevantes". El paso de la traducción por la historia de la Humanidad debe ser discreto, pero no invisible. Es la cantinela de siempre o, como dice Miguel Ángel Vega citando a Valery Larbaud en parecido contexto, el cuento de nunca acabar: el traductor debe conocer su historia para que "sepa de dónde viene y adónde debe ir, para que no repita los mismos errores. Y para que crea en lo que hace, que es hermanar las naciones y las lenguas" (1994: 14).

En efecto, la historia de la traducción ${ }^{1}$ en España ha sido, es y será la "asignatura pendiente" ya no solo entre los saberes que envuelven la formación del traductor, sino también en la historia general. Propiamente, esta denominación metafórica - y harto manida, visto que ya la han utilizado Vega, Lafarga o Payàs para referirse a lo mismo- se convirtió en un término taxativo desde que se llevó a cabo la regulación universitaria de los estudios de traducción, momento en que se dio comienzo a la institucionalización y formalización científica de la disciplina. Ya por entonces, en los 80 y 90, se alzaron algunas de las voces más autorizadas para advertir de la necesidad de historiar la traducción: Antoine Berman (1984: 12-13), Valentín García Yebra (1988: 11), José Lambert (1993), Miguel Ángel Vega Cernuda (1994: 19) o Lieven D'Hulst (1994: 13); pues avanzar en los estudios históricos ayudaría,

1. Pese a que en la tarea de reconstrucción histórica y de escritura historiográfica se dan diferencias sustanciales entre la historia de la interpretación y la historia de la traducción, a menudo ambas disciplinas comparten circunstancias y estudiosos. En el uso general que aquí hacemos del término historia de la traducción entiéndanse incluidas tanto la historia de la práctica como la de la reflexión teórica acerca de la traducción y de la interpretación, tal como se utiliza el término en los títulos de algunos estudios de referencia o incluso a la hora de nombrar las asignaturas que de estas historias se ocupan (cf. Jean Delisle 2003: 221). 
entre otras cosas, a legitimar la traducción como una disciplina independiente, a consolidar la traductología, a introducir al neófito en la disciplina, a promover una mayor tolerancia ante los diferentes enfoques y, a su vez, encontrar la unidad de la disciplina.

No solo se puso de manifiesto la necesidad de historiar la actividad y la reflexión versoras, sino que también fueron surgiendo diversas propuestas metodológicas, como las de Jean Delisle (1996), Brigitte Lépinette (1997), Anthony Pym (1998a), Judith Woodsworth (1998), Samuel López Alcalá (2001), etc., para quienes la aproximación y el análisis de la historia de la traducción debe realizarse siguiendo distintos criterios: para Lépinette, por ejemplo, debe llevarse a cabo atendiendo a lo sociocultural e histórico-descriptivo; para Pym, prestando atención a los traductores y a su espacio intercultural; para López Alcalá, conjugando la recopilación y organización de datos, su análisis e interpretación, y sus estadísticas.

Transcurridas ya unas décadas desde aquel llamado, parece oportuno hacer balance de cuanto se ha producido tanto cualitativa como cuantitativamente. Para medir la producción historiográfica disponemos de los repertorios bibliográficos españoles —ya clásicos- de Fernando Navarro (1996), Julio César Santoyo (1996) y Carmen Valero (2000), cada cual con sus cifras y sus criterios de recopilación. Desde 2001, sin embargo, contamos además con la herramienta BITRA, probablemente la base de datos de bibliografía sobre traducción e interpretación más completa hasta el momento, creada y coordinada por Javier Franco. Algunas de las ventajas de BITRA para la investigación, amén de sus más de 53.000 referencias y de la posibilidad de realizar búsquedas por distintos criterios combinables (año, tema, impacto, tipo de documento, etc.), residen en el hecho de ser una aplicación electrónica que se actualiza constantemente y permite el libre acceso desde Internet. De un modo u otro, cualquiera de estos repertorios viene a confirmar algo ya manifiesto: la eclosión, o mejor, la explosión del fenómeno investigador de la historia de la traducción en los años 80 y el predominio incontestable de los estudios monográficos frente a las obras de conjunto. Para ver más claro ese desarrollo, presentamos a continuación una serie de gráficos realizados a partir de los resultados que arroja BITRA al basar la búsqueda en el tema conjunto de "historia" y "España": 


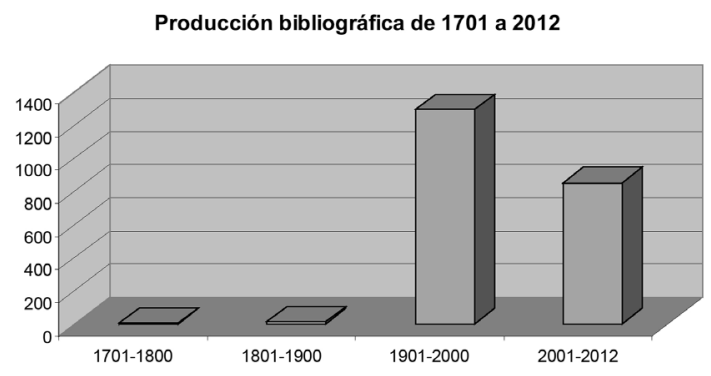

Gráfico 1. Resultados de BITRA de 1701 a 2012

Producción bibliográfica de 1901 a 2012

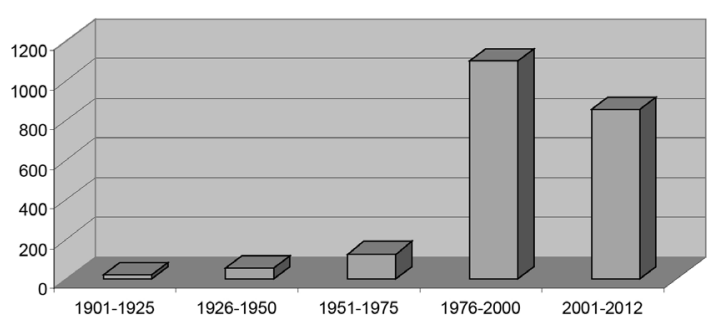

Gráfico 2. Resultados de BITRA de 1901 a 2012

Producción bibliográfica desde 1976

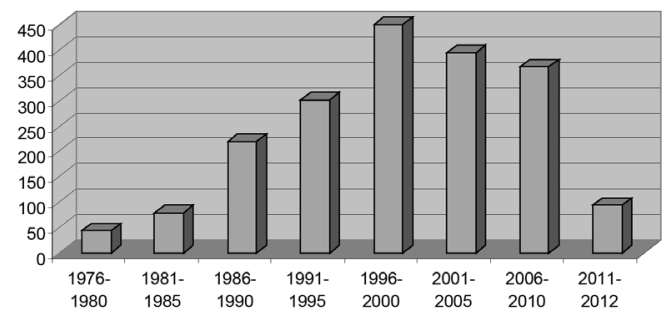

Gráfico 3. Resultados de BITRA de 1976 a 2012

Desde el punto de vista de la cantidad, la situación actual de los estudios sobre la historia de la traducción en España es, en comparación con etapas anteriores, alentadora. No obstante, como hemos apuntado, la mayoría de estos trabajos son capítulos y artículos que aparecen publicados en actas, revistas, monografías, libros, etc. y que abordan aspectos puntuales y temas dispares: versiones de textos clásicos, recepción, traductores, su labor en determinados 


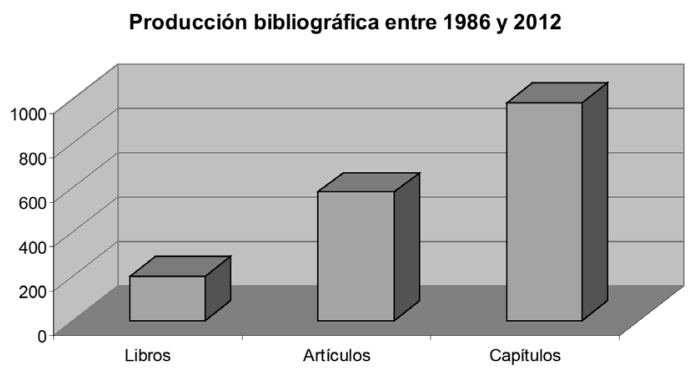

Gráfico 4. Resultados de BITRA entre 1986 y 2012 según el tipo de documento

momentos, etc. Así lo demuestra la diferencia en el número de trabajos publicados durante los años de mayor producción bibliográfica, entre 1986 y 2012 , según sean libros (199), artículos (580) o capítulos (983):

La producción investigadora en historia de la traducción, tan voluminosa como es, adolece de dispersión y falta de sistematización. No obstante, dentro de toda esta historiografía de la traducción hispánica puede trazarse un recorrido por los trabajos más destacados, bien por su valor integrador, bien por su carácter pionero. Por cuestión de espacio, un trabajo de revisión como el presente ha de omitir necesariamente la cita y el comentario de las obras menores, de todos esos capítulos y artículos de revistas que tanto han contribuido al desarrollo historiográfico en España.

\section{Antecedentes historiográficos}

$\mathrm{Al}$ acometer la revisión bibliográfica de la historia de la traducción en España no deja de sorprender que, siendo tan antigua la traducción, su historiación cuente con apenas unas décadas; pues hasta que no se produjo el cambio de paradigma, a mediados de los años 80 del siglo XX, no se comenzó a historiar con el interés y la profundidad que esta disciplina merece. Cierto que las órdenes religiosas realizaban catalogaciones de sus scriptores e, incidentalmente, también de sus traducciones; pero no lo hacían desde la perspectiva traductológica ni mucho menos considerando la traducción — su teoría, su práctica y su historia - como una disciplina con entidad propia. Así, la última parte del Teatro Mexicano (1697), el conocido "Menologio franciscano", del padre Agustín de Vetancurt es quizá el primer trabajo que se refiere expresamente a la actividad de los traductores en el ámbito hispánico. En él recoge el nombre de medio centenar de "varones ilustres", misioneros lingüistas que, como 
indican Vega y Pulido (2012), habrían compuesto y traducido, en ocasiones de manera manuscrita y en otras impresa, obras de carácter lingüístico, litúrgico o pastoral al popoloca, al otomí, al mataltzinca y al náhuatl.

Reseñable es también la iniciativa recopiladora del sacerdote valenciano Josef Joaquín de Lorga, que murió en 1769 recogiendo aún materiales para una Bibliotheca de traductores españoles que nunca vería la luz. Algunas de sus anotaciones las aprovecharía Juan Antonio Pellicer y Saforcada para su conocido trabajo. Mas el meritorio intento de Pellicer, con su Ensayo de una bibliotheca de traductores españoles (1778), se queda igualmente en eso, en un ensayo. Marcelino Menéndez Pelayo hace germinar esa semilla en su Biblioteca de traductores españoles (1874-1878), trabajo ambicioso con el que pretendía suplir una carencia, según explica en Horacio en España (1877: 37): "Doliéndome de que nuestra literatura careciese aún de una Biblioteca de traductores, dejado aparte el ligerísimo ensayo de Pellicer, y perdidos o ignorados los posteriores del P. Bartolomé Pou, de Capmany y de D. Eustaquio Fernández de Navarrete, determiné, tiempo ha, llenar este vacío en cuanto mis fuerzas alcanzasen". La concepción positivista de Menéndez Pelayo acerca de la historiografía fue perentoria, a su vez, para que Cipriano Muñoz y Manzano, conde de la Viñaza, elaborara la célebre Bibliografía española de las lenguas indígenas de América (1892), referencia obligada en el estudio de la labor lingüística de los misioneros traductores en ultramar. Y hasta aquí llegan, después de tantos siglos de intensas relaciones internacionales, los más célebres intentos de historiar la traducción española.

\section{Cambio de paradigma}

Afirma Vega (2004: 18) que fueron Pierre Daniel Huet y Marcelino Menéndez Pelayo quienes pusieron las piedras fundacionales de la disciplina. Huet, con su "De claris interpretibus", publicado en De interpretatione (1683), hilvana la historia de las traducciones bíblicas, analizando detalladamente las versiones de Rufino, Orígenes y san Jerónimo. Como observa Balliu (1995: 47), "la historicidad aparece como la clave del pensamiento hueciano, ya que el enfoque histórico permite poner de manifiesto las traiciones a la verdad original: es de la lengua de la que hay que desconfiar". Por su parte, Menéndez Pelayo, con su Biblioteca de traductores españoles (1874-1878), alienta la concepción de la traducción como disciplina pareja a la literatura, como otro modo de escribir. La obra de José Toribio Medina (1925) acerca de los traductores chilenos es otro de los hitos de esta conciencia historiográfica, que por lo demás sigue la senda abierta por nuestro polígrafo. 
Al hilo de esto, Berman, en la conocida obra que citábamos al principio (1984: 12), explica la importancia capital de adoptar una perspectiva histórica: "La constitution d'une histoire de la traduction est la première tâche d'une théorie moderne de la traduction. À toute modernité appartient, non un regard passéiste, mais un mouvement de rétrospection qui est une saisie de soi". ${ }^{2}$ El germen de esa toma de conciencia que supuso un cambio de paradigma, es decir, el interés moderno por la investigación en historia de la traducción, lo datan Hurtado Albir (2007: 102) y Sabio Pinilla (2006) en 1963, en el IV Congreso de la Federación Internacional de Traductores (FTI), celebrado en Dubrovnik. El aluvión de publicaciones que comenzaron a sucederse desde entonces consolidó los estudios dedicados a la historia de la traducción hasta el punto de que, años después, en el XII congreso internacional de la FTI se creó el Répertoire mondial des historiens de la traduction, y Delisle y Woodsworth publicaron Les traducteurs dans l'histoire (1995). Esta célebre obra colectiva, que contribuyó enormemente a estimular el cambio de mentalidad, marcó asimismo un hito en esa percepción más enfocada de la historiografía general que —al decir de Vega (2004: 19)— "debería utilizar la historia de la comunicación como ciencia auxiliar no solo al lado sino por encima de la numismática o la paleografía".

Con la recepción de las ideas polisistémicas y de las descriptivistas de la Escuela de la Manipulación, los historiadores de la traducción asumirían ahora esa tarea historiadora que, en el ámbito hispánico, solo había ensayado la historia y la filología. Vega Cernuda (2004: 19) retrata del siguiente modo la incuria a la que tradicionalmente venía viéndose abocada la historia de la traducción:

La tarea de historiar la traducción se ve todavía con cierta displicencia, tanto por parte de filólogos como de historiadores. Los puristas de la filología no consideran digna labor propia la crónica de la versión, y la gran historiografía, empeñada en debatir los muertos de la guerra civil española [...] o la talla de estratega del General Franco sin percatarse de que tanto unos como otra tuvieron condicionamientos de comunicación, ve como intrusos a los que levantan acta de esa comunicación.

Aquella displicencia ha quedado ya sepultada por la afluencia de investigaciones que van cubriendo etapas paulatinamente. Los congresos, coloquios, revistas, seminarios y trabajos de historia de la traducción realizados por Jesús Baigorri, Francisco Lafarga, Luis Pegenaute, Julio César Santoyo y Miguel

2. Es decir, "la primera tarea de una teoría moderna de la traducción es reconstruir la historia de la traducción; a la modernidad no le corresponde volver al pasado, sino llevar a cabo un esfuerzo retrospectivo que suponga una toma de conciencia propia". 
Ángel Vega han sido —parafraseando al último de ellos (cf. Vega 2004: 19)— las primeras y angulares piedras sobre las que ha seguido construyéndose la nueva torre de Babel.

\section{Florecimiento historiográfico}

No cabe duda de que los encuentros universitarios han activado y marcado una continuidad en la investigación histórica española, según se desprende del volumen de artículos, comunicaciones, ponencias y publicaciones colectivas que han originado. Al referirnos a los congresos, conferencias y jornadas dedicados a la historia de la traducción debemos citar, en primer lugar, los encuentros de la Universidad de León. Las I Jornadas Nacionales de Historia de la Traducción, celebradas en aquella ciudad en febrero de 1987, dieron el pistoletazo de salida a una constante producción investigadora que perdura en la actualidad. Dejó constancia de aquel encuentro Julio César Santoyo (1988-1989) con los dos volúmenes colectivos de Fidus interpres. Tras dos décadas consagradas a la investigación, este mismo historiador publicaría la valiosa Historia de la traducción: viejos y nuevos apuntes (2008), que recopila varios de sus artículos y conferencias pronunciadas con motivo de diferentes reuniones científicas. El volumen, que se compone de 18 capítulos ordenados cronológicamente desde el siglo III hasta el XX, ofrece un análisis sincrónico del panorama traductológico español en cada momento.

Pese a que en los sucesivos coloquios, congresos y jornadas de distintas universidades españolas por lo común ha venido tratándose la historia de la traducción de forma incidental, destacan por su programa centrado principalmente en aspectos históricos: el coloquio de la Universidad de Granada del que resultó el valioso volumen Traducir la Edad Media (Paredes y Muñoz 1999); los de la Universidad de Barcelona sobre la traducción en España entre 1750-1830 y sobre la traducción entre neoclásicos y románticos (Lafarga 1999; Lafarga et al. 2002); las dos ediciones de las Jornadas HISTRAD celebradas en Alicante, en 2010 y 2012, y dirigidas por Miguel Ángel Vega; el Coloquio Internacional sobre Historia de la Traducción en Hispanoamérica (Barcelona, 2011), así como los ya clásicos Encuentros Complutenses o los Coloquios Internacionales Lucentinos de Traducción, en los que siempre tienen cabida investigaciones de tema histórico. En otros ámbitos de la actividad versora e interpretativa (como el jurídico, el médico, el técnico, etc.) destaca principalmente el calado histórico de los Coloquios Internacionales de Traducción Monacal, celebrados en Guadalupe, en Soria y en Asís entre 2001 y 2011, bajo la codirección de Antonio Bueno y Miguel Ángel Vega. Todas estas reuniones han dado lugar a multitud de artículos recogidos, por lo general, 
en actas y publicaciones colectivas que, si bien no pueden considerarse una fuente primaria por la dispersión de aspectos y de temas históricos que reúnen, sin duda cimientan y consolidan poco a poco la disciplina de la historia de la traducción española.

Junto con lo anterior, la traductografía española cuenta en la actualidad con obras mayores que tratan la historia española de la traducción en su conjunto. Mediante su carácter panorámico, estas obras intentan paliar la falta de sistematización que ha dejado el espontáneo y desordenado florecimiento bibliográfico en el vasto campo de la historia de la traducción. Cronológicamente encontramos en primer lugar la Aproximación a una historia de la traducción en España (2000), de José Francisco Ruiz Casanova, obra pionera en presentar un panorama histórico a lo largo de los siglos. Realiza un estudio sistemático de la historia de la traducción — literaria, sobre todo-, vinculándola con la historia de la literatura y sus grandes periodos cronológicos. Remedando esta obra en lo que respecta a su perspectiva histórico-cronológica, aunque con planteamientos distintos, aparece en 2004 la Historia de la traducción en Espa$\tilde{n} a$, obra colectiva editada por Luis Pegenaute y Francisco Lafarga. El objetivo de esta obra, según sus editores, es presentar adecuadamente, siguiendo un orden cronológico, la situación de la traducción en distintos periodos históricos, combinando las referencias a la actividad traductora con las necesarias alusiones a las poéticas vigentes o generalmente aceptadas en cada periodo. Coincide con la obra anterior en que se centra primordialmente en la historia de la traducción literaria. Con todo, presenta la novedad de tener en cuenta la diversidad lingüística española e incluir, en consecuencia, capítulos específicos sobre la situación en los ámbitos lingüísticos y culturales vasco, gallego y catalán. A este respecto, cabe mencionar la existencia bibliográfica de otros panoramas históricos de la traducción en el País Vasco (Mendiguren 1992), en Galicia (Noia 1995) y en las regiones de lengua catalana (Bacardí y Godayol 2010), redactados en sus respectivas lenguas.

Entre las obras que ofrecen una visión panorámica de la historia de la traducción más allá de lo literario, destacan Interpretación de conferencias: el nacimiento de una profesión, de Jesús Baigorri (1999) e Historia de la traducción en la Administración y en las relaciones internacionales en España (s. XVI-XIX), de Ingrid Cáceres Würsig (2004), quien no pierde la oportunidad en la "Introducción" para denunciar el desequilibrio investigador entre la traducción literaria y la que no lo es. Coincide, pues, con López Alcalá (2001: 105) en que "hay demasiada incidencia en la lingüística, en la lengua, en el texto y en la literatura y poca relevancia para el factor histórico". 
La elaboración de elencos y catálogos de traductores, que como se sabe constituye una tarea con antecedentes pretéritos, retoma su protagonismo con el Diccionario histórico de la traducción en España (2009). La obra, que cuenta con más de 800 entradas, ha sido editada de nuevo por Lafarga y Pegenaute y en ella han participado alrededor de 400 especialistas nacionales e internacionales. Por su parte, en el ámbito lingüístico catalán destaca el Diccionari de la traducció catalana (Bacardí y Godayol 2011), que compila por primera vez a los traductores más importantes de todos los tiempos hasta los nacidos en 1950, que han traducido al catalán. Ha sido realizado por cerca de 80 especialistas y contiene un millar de entradas. Reseñables son asimismo las obras de Alberto Ballestero Izquierdo (1998 y 2007), que abarcan territorios y periodos más restringidos.

Entre los resúmenes sintéticos de la historia de la traducción en España cabe mencionar, en primer lugar, el trabajo de María del Carmen Sánchez Montero redactado en italiano y publicado en 1998. Destaca asimismo el artículo de Van Hoof (1998) escrito en francés y publicado en la revista Hieronymus Complutensis, en el que, como indica Lafarga (2005: 1138), se le cuelan varios "errores de bulto". De otro lado, la Encyclopedia of Translations Studies que edita Routledge dedica en la parte de historia una entrada relativa a España ("Spanish Tradition"). Esta contribución en inglés viene firmada por Anthony Pym en sus dos ediciones (1998b y 2009) y, como en el caso del artículo de Van Hoof, se detiene demasiado en determinados aspectos históricos en detrimento de otros. A la vista de estas aportaciones extranjeras, cabría preguntarse -aunque no lo resolveremos aquí- qué han publicado los historiadores de la traducción españoles en y sobre el exterior.

Las recopilaciones antológicas revisten gran importancia por rescatar para el lector textos de difícil acceso y ofrecer una línea evolutiva de la reflexión sobre la traducción. Se ha reconocido el trabajo de Julio César Santoyo (1987) como el "primer esfuerzo recopilador de textos sobre la traducción en lengua española" (cf. Hurtado Albir 2007: 103). Sobresale destacadamente la antología sobre textos clásicos de teoría de la traducción preparada por Miguel Ángel Vega (1994), quien recorre el discurso traductológico europeo a lo largo de la historia en un amplio y sugerente estudio introductorio, y traza mediante una tabla sinóptica, de forma sintética y muy ilustrativa, la evolución de la traductografía y de la traductología, aunque solo en la primera edición de esta obra. Posteriormente se han sucedido distintas recopilaciones, como la de Dámaso López (1996), la de Francisco Lafarga (1996) o la de Nora Catelli y Marietta Gargatagli (1998). Entre las más recientes, destaca la de Nelson Cartagena (2009), de alto valor histórico por cuanto parte del material 
recolectado permanecía inédito. En un nivel territorial más restringido hallamos, asimismo, estudios antológicos sobre la traducción en el ámbito cultural vasco (Mendiguren 1992), gallego (Dasilva 2003) y catalán (Bacardí, Fontcuberta y Parcerisas 1998; Gallén et al. 2000; García y Lafarga 2004). No puede concluirse este párrafo sin citar la recién publicada obra de J. A. Sabio Pinilla y Pilar Ordóñez (2012), en la que efectúan una revisión del género antológico sobre traducción en los últimos veinticinco años, prestando especial atención a la historia de la traducción.

También se recoge información historiográfica en manuales sobre traducción en general, como los de Eusebio Llácer (1997), Pedro San Ginés (1997) o Amparo Hurtado Albir (2001), quienes dedican una parte sustancial de sus obras al desarrollo histórico de la teoría y de la práctica de la traducción. Asimismo, no pueden olvidarse en este punto las múltiples y valiosas aportaciones de Valentín García Yebra, algunas de ellas recopiladas en sus obras En torno a la traducción (1983) y Traducción: historia y teoría (1994).

Entre los portales web sobre historia de la traducción en España destacan: HISTRAD, que constituye una plataforma abierta para la investigación en historia de la traducción en España e Hispanoamérica, dirigida por Miguel Ángel Vega Cernuda, de la Universidad de Alicante (http://web.ua.es/es/histrad/); la revista electrónica de la historia de la traducción 1611, dirigida por Marietta Gargatagli y Juan Gabriel López Guix, de la Universidad Autónoma de Barcelona (http://www.traduccionliteraria.org/1611/index.htm); la antología de traducciones literarias BITRES, Biblioteca de traducciones españolas, dirigida por Francisco Lafarga y Luis Pegenaute (http://bib.cervantesvirtual. com/portal/bitres/); y el sitio web dedicado a la catalogación y estudio de las traducciones de los franciscanos españoles, que surge del proyecto dirigido por Antonio Bueno, de la Universidad de Valladolid (http://www.traduccionfranciscanos.uva.es/). Por su parte, la Biblioteca Virtual Miguel de Cervantes cuenta con una sección para la "Biblioteca de Traducciones Españolas", dirigida por Francisco Lafarga y Luis Pegenaute en el marco del proyecto BITRES (http://bib.cervantesvirtual.com/portal/bitres/).

En cuanto a las revistas impresas, en la actualidad no existe en el panorama español ninguna que se ocupe íntegramente de temas relacionados con la historia de traducción, sea en su vertiente práctica, sea en la teórica. Las que más atención han prestado a los aspectos históricos son, por orden cronológico: la revista Livius, del Departamento de Filología Moderna de la Universidad de León, que editó catorce números entre 1992 y 1999; Hieronymus Complutensis, del Instituto de Universitario de Lenguas Modernas y Traductores, de la Universidad Complutense, que ha editado doce tomos desde 
1995; y Hermēneus, de la Facultad de Traducción e Interpretación de Soria, que comenzó a editarse en 1999.

Aunque en otro rango de análisis, es justo recordar cómo el ámbito académico ha jugado un importante papel en la promoción de la investigación sobre la historia de la traducción. Como es sabido, en 1974 se creó el Instituto Universitario de Lenguas Modernas y Traductores (IULMyT) de la Universidad Complutense de Madrid, convirtiéndose en el primer centro oficial con rango plenamente universitario dedicado al estudio y la investigación de la traducción en España. La importancia del IULMyT radica, respecto del tema que nos atañe, en el hecho de ser la primera institución española donde se impartió, en el marco del primer programa de doctorado en traducción en 1991, la asignatura de historia de la traducción, cuyo responsable fue Miguel Ángel Vega Cernuda. La primera tesis doctoral surgida de aquel curso de historia de la traducción impartido en España fue escrita por Lidia Taillefer de Haya a propuesta y bajo la dirección del mismo Miguel Ángel Vega. Se leyó en 1995 y llevaba por título Bibliografía anglosajona de la traducción y ciencias auxiliares: corpus y examen crítico.

\section{La historia de la traducción en las aulas: perspectivas de futuro}

La revisión de la bibliografía sobre la historia de la traducción en España constituye una buena forma de observar la evolución del interés que esta materia ha suscitado. E igual de revelador puede ser el examen de los actuales planes de estudio del grado en traducción. Sin embargo, lo que se constata es bien distinto: aquel interés investigador del que ha resultado tanta bibliografía aún no tiene correspondencia en el ámbito docente, donde se relativiza su importancia. Baste decir al respecto que de las 24 universidades españolas que imparten el grado en traducción, tan solo seis (es decir, el $25 \%$ ) incluyen en sus planes de estudio la asignatura de historia de la traducción. Pese a su introducción paulatina, su aceptación —no digamos ya su interés— entre el alumnado resulta de todo punto cuestionable, visto que sienten la imperante necesidad de centrar sus conocimientos en las tecnologías de la traducción, es decir, en meras herramientas puestas al servicio de la traducción para dar solución a las nuevas exigencias tecnológicas. Según se observa, la mayoría de los estudios de grado están enmarcados en el triángulo universidad-alumnoempresa. En tal contexto se va imponiendo, y aun entronizando, en aras de la productividad — que no precisamente de la calidad — el aprendizaje de programas de traducción asistida por ordenador, saber tan necesario y accesorio como en su día la mecanografía o la ofimática, cuyos meros propósitos productivistas seducen a la mayor parte de un alumnado preocupado, evidente 
y únicamente, por ganarse la vida traduciendo. Muestra de ello es el éxito, en términos de demanda, que tienen los másteres y cursos de localización y tradumática, frente a los de corte puramente humanístico.

De entre esas seis universidades que ofertan una asignatura de grado dedicada a la historia de la traducción, algunas imparten esta materia como parte sustancial de una asignatura más amplia dedicada a la teoría de la traducción. Así, por ejemplo, la asignatura Teoría e Historia de la Traducción que se ha impartido en la Universidad de Alicante (curso 2011-2012) combina la exposición de los contenidos con el análisis de textos, los cuales pueden ser fuentes teóricas fundamentales o traducciones que reflejen la teoría vista en clase. Respecto de su vertiente histórica, contempla los siguientes contenidos: pensadores y escuelas de la teoría clásica de la traducción, de Cicerón a Ortega y Gasset; pensadores y escuelas de la teoría moderna de la traducción: de un lado, lingüística contrastiva, pragmática y de corpus; de otro, las escuelas funcionalistas (escopo y polisistemas), y de otro, el giro ideológico (crítica postcolonialista, feminista y visibilidad del traductor); la traducción y los traductores en la historia universal, la historia de la traducción en España hasta la Segunda República y su historia desde entonces hasta la actualidad. De este modo, la asignatura pretende: a) dotar al futuro traductor de las bases reflexivas que le capaciten para un ejercicio consciente, racional y, al mismo tiempo, creativo de la disciplina; b) sentar las bases para el futuro interés investigador por los problemas operativos, culturales y antropológicos de la traducción; c) transmitir el conocimiento y la reflexión crítica de las principales escuelas que han aglutinado el pensamiento lingüístico, cultural y antropológico sobre la traducción, tanto desde el punto de vista histórico como desde el sistemático; y d) capacitar para que el alumno desarrolle una conciencia profesional y crítica sobre el ejercicio de la propia actividad.

\section{Conclusiones}

El recorrido efectuado nos lleva a extraer algunas conclusiones y plantear ciertas conjeturas. De un lado, queda patente el incremento que han experimentado los estudios sobre la historia de la traducción en España a raíz del cambio de paradigma que supuso la adopción de las teorías descriptivistas y del polisistema. De otro lado, coincidiendo con Lafarga (2005: 1134), se pone de manifiesto que ese florecimiento investigador, esa abundancia, a menudo se traduce en una inmensa variedad e, incluso, dispersión. El grueso de la producción bibliográfica lo constituyen capítulos y artículos frecuentemente dispersos en diversos canales de difusión, que, pese a no consagrarse íntegramente a aspectos históricos, sin duda han sentado las bases para un mejor 
conocimiento - aunque todavía insuficiente— de la historia de la traducción y de la reflexión teórica en español.

La dispersión de la que hablamos también ha planteado la necesidad práctica de elaborar obras que ofrezcan una visión panorámica de la historia de la traducción. En este sentido, ha habido que esperar hasta el año 2000 para que apareciera la primera obra de conjunto, a la que le ha seguido otra más ambiciosa, en 2004, que igualmente encalla ante la problemática de determinar los periodos propios de la traducción, y sigue los criterios de la historiografía de la literatura o la historia cultural españolas. Definir los periodos y las clasificaciones traductológicas para una obra de síntesis que fuera más allá de la traducción literaria, sin duda constituye un auténtico nudo gordiano (cf. Zarrouk 2007: 126). Su resolución, sin embargo, contribuiría de forma perentoria a dotar de solidez metodológica a la investigación histórica y la reflexión teórica sobre la traducción.

Como apuntábamos al principio, ya son muchos los autores que exigen historiar la traducción partiendo de una fundamentación metodológica. Vega Cernuda (1996: 85) ya mostró que "de nada nos sirven los cuadros históricos, si no es, como dijo el clásico al llamar a la historia magistra vitae, para sacar consecuencias prácticas. Este es el horizonte de toda historiografía traductora: evitar errores no solo de procedimiento, sino también de apreciación mutua". En lo mismo incide López Alcalá, quien, apoyando las posturas de Jean Delisle y Anthony Pym, sostiene que la historia de la traducción carece aún de solidez metodológica y que se hace imprescindible una aproximación historiográfica de esta disciplina, donde la propia historia debe ocupar un lugar de honor (López Alcalá 2001; citado en Cáceres 2004: 22). En época más reciente han reanimado el debate sobre la metodología, entre otros, Sabio Pinilla (2006), Mourad Zarrouk (2006 y 2007) y, de nuevo, Vega Cernuda (en el presente número de MonTI). Lo cierto es que, ante el desarrollo que han experimentado los estudios sobre la historia de la traducción, resulta paradójico que todavía no se haya establecido una periodificación ni una clasificación consensuada que siente las bases de la teoría historiográfica de la traducción en España y permita adoptar una perspectiva sintética sobre la historia.

Por otro lado, el repaso bibliográfico deja claro, además, que el interés entre los historiadores de la traducción (con independencia del territorio y de la lengua peninsular a los que se refieran) se ha manifestado principalmente por la historia de la traducción literaria, dejando de lado la de otros ámbitos no menos importantes en el devenir de la historia, como son la traducción jurídico-administrativa, la traducción económica, la traducción científico-técnica, la traducción médica, etc. Las obras de conjunto lo son desde el punto de vista 
cronológico, pero no tanto desde una perspectiva integradora de las distintas actividades versoras e interpretativas.

Por último, si bien en el ámbito de la investigación las perspectivas de recuperación histórica son ciertamente alentadoras, no puede decirse lo mismo para el ámbito docente. Abríamos este trabajo refiriéndonos a la historia de la traducción como asignatura pendiente en el pasado, en el presente y en el futuro. En el pasado, porque hasta mediados de los 80 no se produjo el cambio de paradigma por el que se entendería la traducción como disciplina independiente. En el presente, porque, a pesar de la multitud de estudios que abordan la traducción desde una perspectiva histórica, aún no se ha consensuado una periodificación ni una clasificación de la historiografía traductológica, de modo que seguimos careciendo de obras de compendio que estudien de forma sistemática la historia de la traducción en todas sus facetas. Y en el futuro, porque la Universidad ha demostrado su incapacidad para ser autónoma — que no ajena— ante las coyunturas económicas y, con ello, un notorio desdén por las disciplinas humanísticas en pro de las técnicas. En los planes de estudios cuyo diseño se ha orientado a objetivos mercantilistas, las enseñanzas técnicas de la profesión podrían acabar desbancando la asignatura de historia, que en el mejor de los casos quedaría relegada a los estudios humanísticos de posgrado, justo donde empezó a impartirse. Poco nos habría enseñado entonces toda esta historia en caso de darse tal desubstanciación del cometido humanístico del traductor.

\section{Bibliografía}

BAIGORRI, Jesús. (1999) Interpretación de conferencias: el nacimiento de una profesión. Granada: Comares.

Ballestero IzQUierdo, Alberto. (1998) Diccionario de traducción: traducciones y traductores en Navarra, (siglos XV-XIX). Pamplona: Eunate.

BALlestero IzQUiERDO, Alberto. (2007) Traducción y traductores de entreguerras (1918-1936). Pamplona: Eunate.

BALliU, Christian. (1995) "Los traductores transparentes: Historia de la traducción en Francia durante el período clásico." Hieronymus Complutensis 1, pp. 9-51.

BACARDí, Montserrat; Joan Fontcuberta \& Francesc Parcerisas (eds.) (1998) Cent anys de traducció al catalá (1891-1990). Barcelona: Eumo.

BACARDí, Montserrat \& Pilar Godayol. (2010) Una impossibilitat possible. Trenta anys de traducció als Països Catalans (1975-2010). Vilanova i la Geltrú: El Cep i la Nansa. 
BACARDí, Montserrat \& Pilar Godayol. (2011) Diccionari de la traducció catalana. Vic: EUMO.

Berman, Antoine. (1984) L'épreuve de l'étranger. París: Gallimard. (Trad. española: La prueba de lo ajeno. Las Palmas: Universidad de Las Palmas de Gran Canaria).

BITRA (Bibliografía de Interpretación y Traducción): Base de datos bibliográfica creada en 2001 por Javier Franco Aixelá, de la Universidad de Alicante. En línea: <http://dti.ua.es/es/bitra/introduccion.html>

CÁCERES WÜRSIG, Ingrid. (2004) Historia de la traducción en la Administración y en las relaciones internacionales en España. Soria: Vertere, monográficos de la revista Hermēneus.

CARTAGENA, Nelson. (2009) La contribución de España a la teoría de la traducción. Introducción al estudio y antología de textos de los siglos XIV y XV. Madrid/ Frankfurt: Iberoamericana/Vervuert.

Catelli, Nora \& Marietta Gargatagli (eds.) (1998) El tabaco que fumaba Plinio. Escenas de la traducción en España y América. Barcelona: Edicions del Serbal.

Dasilva, X. M. (2003) Babel entre nós. Escolma de textos sobre a traducción en Galicia. Vigo: Universidade de Vigo.

Delisle, Jean. (1996) "Reflexións sobre as esixencias científicas da historia da traducción." Viceversa 2. pp. 37-56; reproducido en "Réflexions sur l'historiographie de la traduction et ses exigentes scientifiques." Équivalences 26:2-27:1, pp. 21-43.

DELISLE, Jean. (2003) "La historia de la traducción y su importancia para la traductología y su enseñanza mediante un programa didáctico multimedia y multilingüe." Íkala, revista de lenguaje y cultura, 8, núm. 14, pp. 221-235.

DELISLE, Jean et Judith Woodsworth. (1995) Les traducteurs dans l'histoire. Ottawa: Presses de l'Université d'Ottawa.

D'Hulst, Lieven. (1994) "Enseigner la traductologie: pour qui et à quelles fins?" Meta 39:1, pp. 8-14.

GALlÉN, E. et al. (2000) L'art de traduir. Reflexions sobre la traducció al llarg de la història. Vic: Eumo.

García Yebra, Valentín. (1988) "Protohistoria de la traducción." En: Julio César Santoyo \& al. (ed.) 1988. Fidus interpres. Actas de las I Jornadas Nacionales de Historia de la Traducción. León: Universidad de León, I, pp. 11-23.

García Yebra, Valentín. (1983) En torno a la traducción. Teoría. Crítica. Historia. Madrid: Gredos.

García Yebra, Valentín. (1994) Traducción: historia y teoría. Madrid: Gredos.

García Garrosa, M. J. \& Francisco Lafarga. (2004) El discurso sobre la traducción en la España del siglo XVIII. Estudio y antología. Kassel: Reichenberger.

Hoof, Henri van. (1998) "Esquisse pour une histoire de la traduction en Espagne." Hieronymus Complutensis 6-7, pp. 9-23. 
HuET, Pierre Daniel. (1683) De interpretatione Libri duo, quorum prior est de optimo genere interpretandi; alter de claris interpretibus. La Haya.

HurTAdo AlBIR, Amparo. (2001) Traducción y Traductología. Introducción a la Traductología. Madrid: Cátedra. [2ª Ed. 2007].

LAFARGA, Francisco (ed.) (1996) El discurso sobre la traducción en la historia (antología bilingüe). Barcelona: EUB.

LAFARGA, Francisco. (2005) "Sobre la historia de la traducción en España: contextos, métodos, realizaciones." Meta 50:4, pp. 1133-1147.

LAFARGA, Francisco (ed.) (1999) La traducción en España, 1750-1830. Lengua, literatura, cultura. Lleida: Universitat de Lleida.

LAfArga, Francisco; Concepción Palacios \& Alfonso Saura (eds.) (2002) Neoclásicos y románticos ante la traducción. Murcia: Universidad de Murcia.

Lafarga, Francisco \& Luis Pegenaute (eds.) (2009) Diccionario histórico de la traducción en España. Madrid: Gredos.

LAfarga, Francisco \& Luis Pegenaute (eds.) (2004) Historia de la Traducción en España. Salamanca: Ambos Mundos.

LAMBERT, José. (1993) "History, Historiography and the Discipline. A Programme." En: Gambier, Yves \& Jorma Tommola (eds.) 1993. Translation $\mathcal{E}$ Knowledge. Turku: University of Turku, pp. 3-26 ("Scandinavian Symposium of Translation Theory" 4).

LÉPINETTE, Brigitte. (1997) La historia de la traducción. Metodología. Apuntes bibliográficos. Valencia: Centro de Estudios sobre Comunicación Interlingüística e Intercultural ("LynX. Documentos de trabajo" 14).

LLÁCER, Eusebio V. (1997) Introducción a los estudios sobre traducción. Historia, teoría y análisis descriptivos. Valencia: Depto. de Filología Inglesa y Alemana ("Cuadernos de Filología. Anejos" XX).

López García, Dámaso. (1996) Teorías de la traducción: Antología de textos. Cuenca: Universidad de Castilla la Mancha.

López Alcalá, Samuel. (2001) La historia, la traducción y el control del pasado. Madrid: Universidad Pontificia de Comillas.

Mendiguren, Xabier. (1992) Itzulpengintza: historia eta teoria. Donostia: Elkar.

MenÉndez Pelayo, Marcelino. (1874-1878) Biblioteca de traductores españoles. Madrid: Aldus \& CSIC. [Ed. de 1952].

MenÉndez Pelayo, Marcelino. (1877) Horacio en España. Madrid: Casa Editorial de Medina.

Muñoz y Manzano, Cipriano (conde de la Viñaza). (1892) Bibliografía española de lenguas indígenas de América. Madrid: Est. tipográfico Sucesores de Rivadeneyra. [Ed. facsimilar, Madrid: Atlas, 1977].

NAVArRo Domínguez, Fernando. (1996) Manual de bibliografía española de traducción e interpretación. Diez años de historia: 1985-1995. Alicante: Universidad de Alicante. 
Noia CAmpos, Ma Camino. (1995) "Historia da traducción en Galicia no marco da cultura europea." Viceversa 1, pp. 13-62.

PAREDES, Juan \& Eva Muñoz (eds.) (1999) Traducir la Edad Media. La traducción de la literatura medieval románica, Granada: Universidad de Granada.

PELlicer y SAForCADA, Juan Antonio. (1778) Ensayo de una biblioteca de traductores españoles. Madrid: Antonio de Sancha. [Reprod. facsímil, Cáceres: Universidad de Extremadura, Servicio de Publicaciones, 2002].

PyM, Anthony. (1998a) Method in Translation History. Manchester: St. Jerome Publishing.

PyM, Anthony. (1998b) "Spanish Tradition." En: Baker, Mona (ed.) 1998. Routledge Encyclopedia of Translation Studies. London/New York: Routledge, pp. 552-563

PyM, Anthony. (2009) "Spanish Tradition.” En: Baker, Mona \& Gabriela Saldanha (eds.) 2009. Routledge Encyclopedia of Translation Studies. Nueva York: Routledge, pp. 533-542.

Ruiz Casanova, José Francisco. (2000) Aproximación a una historia de la traducción en España. Madrid: Cátedra.

SABio Pinilla, José Antonio. (2006) "La metodología en historia de la traducción: estado de la cuestión.” Sendebar 17, pp. 21-48.

Sabio Pinilla, José Antonio \& Pilar Ordóñez López. (2012) Las antologías sobre la traducción en el ámbito peninsular. Análisis y estudio. Berna: Peter Lang.

SAN GINÉS, Pedro. (1997) Traducción teórica. Planteamientos generales y teóricos de la traducción. Granada: Comares.

SÁnCHEZ Montero, María del Carmen. (1998) Lineamenti di storia della traduzione in Spagna. Trieste: Università degli Studi di Trieste.

SAntoyo, Julio César. (1987) Teoría y práctica de la traducción. Antología. Bellaterra: EUTI-Universitat Autònoma de Barcelona.

SAntoyo, Julio César et al. (ed.) (1988-1989) Fidus interpres. Actas de las I Jornadas nacionales de historia de la traducción. 2 vols. León: Universidad de León.

SANTOYO, Julio César. (1996) Bibliografía de la traducción en español, catalán, gallego y vasco. León: Universidad de León.

SANTOYO, Julio César. (2008) Historia de la traducción: viejos y nuevos apuntes. León: Universidad de León.

TAILlEFER DE HAYA, Lidia. (1995) Bibliografía anglosajona de la traducción y ciencias auxiliares: corpus y examen crítico. Tesis doctoral. Madrid: Universidad Complutense-IULMyT. Versión electrónica publicada en 2006 bajo el título Traductografía y traductología en lengua inglesa: <http://traductolog.filosofia. uma.es/ediciones.html>

Toribio Medina, José. (1925) Bibliotecha chilena de traductores (1820-1924). Santiago: Soc. Imprenta y Litografía Universo. 
VAlero Garcés, Carmen. (2000) "Translating as an Academic and Professional Activity." Meta 45: 2, pp. 379-383.

Vega Cernuda, Miguel Ángel. (ed.) (1994) Textos clásicos de teoría de la traducción. Madrid: Cátedra. [2. ${ }^{a}$ Edición en 2004].

Vega CernudA, Miguel Ángel. (1996-1997) "Apuntes socioculturales de Historia de la Traducción: Del Renacimiento hasta nuestros días." Hieronymus Complutensis 4-5, pp. 71-85.

Vega CERnUdA, Miguel Ángel. (2004) "Presentación: la historia como imperativo moral." Prólogo a Cáceres Würsig, Ingrid 2004. Historia de la traducción en la Administración y en las relaciones internacionales en España. Soria: Vertere, monográficos de la revista Hermēneus, pp. 17-19.

VetancurT, fray Agustín de, OFM. (1697) Teatro Mexicano: descripción breve de los sucesos ejemplares, históricos y religiosos del Nuevo Mundo de las Indias. Crónica de la Provincia del Santo Evangelio de México. Menologio franciscano de los varones más señalados, que con sus vidas ejemplares, perfección religiosa, ciencia, predicación evangélica en su vida, ilustraron la Provincia del Santo Evangelio de México. México: Editorial Porrúa, 1971.

WoODSWORTH, Judith. (1998) "History of translation.” En: Baker, Mona (ed.) Routledge Encyclopedia of Translation Studies. London/New York: Routledge, pp. 100-106.

ZARROUK, Mourad. (2006) "Microhistoria e historia de la traducción." Sendebar 17, pp. 5-19.

ZARrouk, Mourad. (2007) "Hacia otra Historia de la Traducción." En: Navarro, Fernando et al. (eds.) 2007. La traducción: balance del pasado y retos del futuro. Alicante: Aguaclara y Dpto. de Traducción e Interpretación de la Universidad de Alicante. 


\section{NOTA BIOGRÁFICA / BIONOTE}

David Pérez Blázquez posee una Licenciatura en Traducción e Interpretación, acompañada de una selección de asignaturas de Derecho, y el Máster Oficial en Traducción Institucional por la Universidad de Alicante, así como el título de traductor-intérprete jurado por el Ministerio de Asuntos Exteriores y Cooperación español. Ejerce profesionalmente como traductor autónomo y dedica gran parte de su tiempo al estudio de la historia de la traducción. Desde 2006 colabora con carácter honorífico con el grupo de investigación Histrad, dirigido por Miguel Ángel Vega Cernuda. Su producción investigadora se centra sobre todo en temas relacionados con la recepción literaria, la historia y la historiografía de la traducción, especialmente en los ámbitos alemán e hispanoamericano.

David Pérez Blázquez holds a degree in Translation and Interpretation, supplemented with a selection of subjects of Law, and a MA in Translation from the University of Alicante, as well as the accreditation of sworn translator issued by the Spanish Foreign Affairs and Cooperation Ministry. Nowadays, he is working as a freelance translator and devotes much of his time to studying the history of translation. Since 2006, he is an honorary member of the research group Histrad, led by Prof. Miguel Ángel Vega Cernuda. His research production focuses primarily on issues related to the reception of literature, the history and the historiography of translation, especially in the German and Latin American areas. 